\title{
An autonomous navigational system using GPS and computer vision for futuristic road traffic
}

\author{
Prabha Ramasamy ${ }^{1,2}$, Mohan Kabadi ${ }^{3}$ \\ ${ }^{1}$ Department of Computer Science and Engineering, Sai Vidya Institute of Technology, Bangalore, India \\ ${ }^{2}$ Department of Computer Science and Engineering, Visvesvaraya Technological University, Belagavi, India \\ ${ }^{3}$ Department of Computer Science and Engineering, Presidency University, Bangalore, India
}

\begin{tabular}{l} 
Article Info \\
\hline Article history: \\
Received Jul 14, 2020 \\
Revised Jun 3, 2021 \\
Accepted Jul 1, 2021 \\
\hline
\end{tabular}

Keywords:

Accuracy

Computer vision

Geo-reference data

Global positioning system

Visual sensor

\begin{abstract}
Navigational service is one of the most essential dependency towards any transport system and at present, there are various revolutionary approaches that has contributed towards its improvement. This paper has reviewed the global positioning system (GPS) and computer vision based navigational system and found that there is a large gap between the actual demand of navigation and what currently exists. Therefore, the proposed study discusses about a novel framework of an autonomous navigation system that uses GPS as well as computer vision considering the case study of futuristic road traffic system. An analytical model is built up where the geo-referenced data from GPS is integrated with the signals captured from the visual sensors are considered to implement this concept. The simulated outcome of the study shows that proposed study offers enhanced accuracy as well as faster processing in contrast to existing approaches.
\end{abstract}

This is an open access article under the CC BY-SA license.

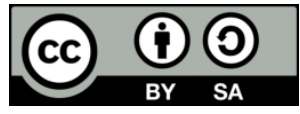

\section{Corresponding Author:}

Prabha Ramasamy

Department of Computer Science and Engineering, Visvesvaraya Technological University

Belagavi, Karnataka, India

Email: research.prabha.r@gmail.com

\section{INTRODUCTION}

Basically, a navigation system can be considered to be a sophisticated computing mechanism that facilitates in offering navigational services [1]. A typical navigation system consists of a geographic information in the form of a map that offers user friendly format of reading geographic data often in the form of text. They can also use various forms of sensor and other sources of information collected from different ways. At present, they are used for recommending suitable direction of the routes for the vehicles where the navigational device is installed. There are various forms of navigational system viz. deep space navigation, robotic mapping, inertial navigation system, surgical navigation system (used in medical sector), satellite navigation system, and automotive navigational system [2], [3]. Out of all the forms of navigational system, global positioning system (GPS) is the most adopted way among the global users. GPS uses a radionavigation mechanism that offers information about the time and geo-location to the receiver of GPS from any point of the earth. There are various reasons for higher technical adoption of GPS as it is completely independent of any data to be fed by the user and it can operate on its own [4]. It does not have even a dependency of internet reception or any telephonic services to be functional although using these services offers more accuracy in navigation. A typical application of GPS based navigational services emphasizes on civilians, time-keeping, communication, and military applications [5]. However, there are certain issues associated with the GPS usage i.e.; i) it is not capable of monitoring condition of the vehicle or object where it resides as receiver, ii) it often encounters outage issues owing to signal attenuation, iii) it often encounters 
issues for deviation alters in real-time, and iv) information obtained from it are not precise always [6]. In this regards, there has been various research work being carried out to overcome these problems; however, the legacy problem remains the same more or less. Hence, GPS is often used in integration of the inertial navigation system [7]. However, there are still issues in all these on way around. Along with this consideration, it is required to also accept that modern day's communication system in road traffic system is also undergoing revolution. The concepts of vehicular ad hoc network with integration of internet-of-things are now future of modern road traffic system [8]. In this system, an embedded device called as on-board units resides in the vehicle which communicates with the hotspot called as road-side unit on the road and it allows a distributed vehicular communication system. As on-board units are also connected with the infotainment system which has accessibility to GPS based navigational system, there is a fair chance that an autonomous and wireless navigational system can be designed which can actually control the navigation in much better way. Apart from this, there is already IP-based surveillance camera which is mounted on road. If this surveillance cameras are exchanged with cost effective visual sensors than a novel form of navigation system using computer vision. There is a good possibility that usage of computer vision can offer more precise information about the mobile object i.e., vehicle which can be integrated with GPS data to offer more accurate navigation system.

Therefore, the proposed system introduces a novel approach where computer vision and GPS mechanism is used for framing up a cost effective navigational system. The paper also highlights the approach and its complete operation with the help of three simple and efficient algorithms assisting in navigation. The organization of this paper is as follows: Section 1 discusses about the existing navigational approaches concerning about GPS and computer vision, identified research problem, and followed by proposed solution. Section 2 discuss about proposed method, while research method is discussed in section 3. Section 3.1 discusses about the algorithm implementation. The analysis of the obtained results is discussed in Section 4, while the concluding remarks are highlighted in section 5.

The proposed solution our prior work has discussed about the existing approaches towards enhancing the performance of navigation system [9]. This section further updates about more length of work being carried out most recently in similar domain of research work. It has been noticed that GPS has been always a dominant contributor towards navigational system. The recent work carried out by Abosekeen et al. [10] has presented a model where azimuth angle is emphasized towards the precise routing process using a unique pre-processing operation as well as update-based operation towards received signal strength during the outage of the GPS data. Recent studies have also been carried out towards unmanned aerial vehicle system where both GPS as well as inertial navigation system (INS) has been used. The work carried out by Guo et al. [11] has used a unique model for identifying and isolating the counterfeited signal from the genuine version of the navigational signal. The work carried out by Li et al. [12] have used extended Kalman filter using both GPS as well INS with an idea to control the artifacts present dues to attitude error. The paper contributes towards parameterizing the attitude error. A joint implementation of GPS along with Doppler velocity log and INS as seen in work reported by Mostafa et al. [13] using a filter mechanism to control accuracies. The works of Steiner et al. [14] have investigated the usage of GPS in snow area which offers an influence. It also assists in isolating the signals inflicted with interference. The work carried out by Wang et al. [15] have used neural network for the purpose of developing a model that can solve the outage issues in GPS/INS system. Nearly similar problem has also been addressed by Zhang et al. [16]; however, the authors have used a filtering approach as well as neural network with short term memory approach in order to offer information when there is an outage of GPS information. Li et al. [17] have carried out another similar form of study where the joint usage of GPS and INS was used for navigation system with Kalman filter for higher degree of accuracy in navigation system in case of outage of GPS. The work carried out by Guo et al. [18] has presented a navigational model.

Apart from GPS-based approach, modern literatures are also found to use an off-the grid method for navigational system. In this approach, usage of computer vision has been witnessed. The work carried out by Dong et al. [19] has developed a navigation system on the basis of the information derived from the sensors inbuilt within a smart phone. This technique develops a three-dimensional navigational model for indoor area. The study is found to offer almost instantaneous localization of user with less error. Hardware-based experimentation using similar approach of navigation system using computer vision is witnessed in the work of Lentaris et al. [20]. The authors have used field programmable gate array (FPGA) with highly customized design where the outcome shows highly reduced errors. The work carried out by Manzanilla et al. [21] has also used computer vision for performing navigation of unmanned vehicle system. The authors have used Kalman filter for improving the estimation of the pose using the experimental model of robotics. There are various associated studies where computer vision has been used for navigation application [22]-[30]. Next, discusses about the identified research problems that has been not addressed yet. 
The identified research problem has been explored after reviewing the existing approaches using GPS as well as computer vision approach. This section highlights the open end research problems associated with the navigational factor: i) there is no research work to consider the mechanism defects of device which executes INS system; ii) there are no such literatures using geo-referenced data for different format of nonGPS data; iii) existing methods offers restrictions as the scope of the algorithms are limited to only the presented scenario as well as dataset; iv) existing approaches of computer vision are actually not applicable in modern transportation system; and v) there is an absence of any standard navigational system either using GPS or using computer vision. Therefore, after reviewing the above mentioned open end research issues, the proposed system crafts a statement of problem as "it is challenging to develop a cost effective and efficient navigational system by joint implementation of GPS and computer vision". Next, discusses about proposed solution and its methodologies adopted.

The primary idea of the proposed system is therefore to offer an integration of the computer vision as well as GPS data in order to carry out an effective navigation system. At present, there are many such trends observed where the navigation system is developed with an aid of different forms of imageries. Apart from this, traffic based real-time imageries offer highly informative data from various sensors as they can be captured from the much closed range in comparison to the GPS data. Hence, a better granularity in the traffic information can be obtained in this perspective.

\section{THE PROPOSED METHOD}

The proposed system is an extension of our prior implementation where GPS has been used for navigational system [31]. The present implementation is further an improved version where a novel system model is designed and developed for assisting in navigational system of modern day's traffic. An analytical model is presented in proposed system which targets towards. After reviewing the existing approaches associated with the navigational system, it is found that GPS is very much an integral part of the mechanism. At the same time, it is essential to know that availability of the GPS data is not always guaranteed. Therefore, the proposed system has an inclusion of an additional data which is the visual signals captured from the visual sensor hosted in the specific traffic spots. Figure 1 shows an intersection of 4 lane which is equipped with visual sensors that are hosted on the road-side units. Considering that a smart vehicular network is running on the road which will mean that on-board unit of all the vehicles are connected with the road-side units and all the 4 road-side units are connected to a centralized traffic gateway at the end.
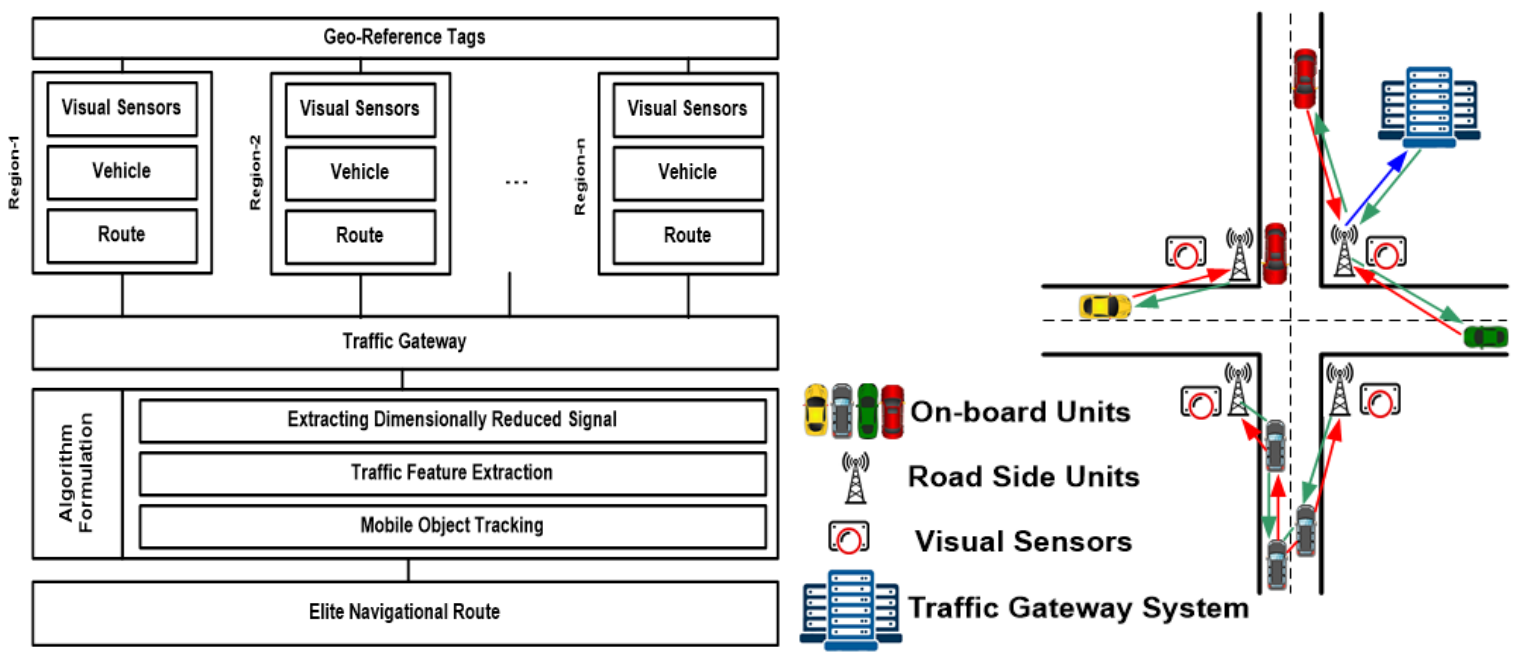

Figure 1. Proposed system

The study assumes that traffic-gateway system is responsible for three operations viz, i) accepting the data generated by the road-side units, ii) applying algorithm over the data to find out the number of vehicles running on each lane, their respective position, and their direction, and iii) transmitting back the outcome to the road-side units. The road-side units further forward the data to its vehicle within the range and they vehicle will be offered a new navigational scenario. The drivers can maneuver their vehicles with that information. Hence, in this perspective there are two things required to be adhered on viz, i) accuracy in 
positioning and ii) faster relay of navigational filtered data. This approach towards navigation has never been adopted in past and hence it contributes towards a novel cost-effective navigational system for futuristic transport system. This algorithm further offers dual advantage viz. i) size of data reduces down further as features of signal are more reduced in size compared to original signal, ii) it offers more precise information which can narrow down the search for better navigational yield. The final algorithm is responsible for further tracking all the objects that performs mobility. This algorithm offers following advantage viz, i) it can perform tracking over the streaming data and hence resultant is almost instantaneous, ii) it can track down all the objects present in the scene while static or mobile, and iii) it can offer precise count of the vehicles present on the scene to offer better insight in decision making. Hence, the proposed algorithm has no such inclusion of any process which is sophisticated, time-consuming, and iterative. Therefore, a cost effective solution towards navigational system by combining GPS and computer vision is presented.

\section{RESEARCH METHOD}

Basically, the proposed system also makes use geo-referencing process which is basically a mechanism of tagging ground coordinate system of the moving objects. Different geographic regions have mounted with the road-side units, which consist of visual sensors, information about mobile vehicles, and route information stored in its memory system. All this information is subjected to aggregation and is then forwarded to the traffic gateway system. After this information is collected, the next step will be to apply an algorithm to this set of traffic information. Considering a case where the road-side units are using cloudbased services to upload the information to traffic gateway, the applicability of proposed system will look more practical. As this stream of information is quite high in number, therefore, the first task of the proposed system will be to minimize the original dimensionality of this information. This is because the outcome of the algorithm processing is basically a direction navigational information based on which the topology of the movement of the vehicle will be amended based on wish of the driver. Hence, this information should travel the network in faster rate and hence the first algorithm will assist to overcome this problem. The filtered outcome of the first algorithm is then subjected to the second algorithm which is responsible for extracting significant features from the dimensionally reduced traffic data in prior step.

\subsection{Algorithm implementation}

This section discusses about the algorithm that has been implemented towards achieving the goal of the proposed system.

\subsubsection{Algorithm for extracting dimensionally reduced signal}

Prior to discussion of this algorithm, it is require stating that this algorithm works on the assumption that there are visual sensors that are grounded on various traffic position. The signals that are captured from the visual sensors at a specific interval rate is forwarded to a traffic gateway system which runs the proposed algorithm. It will be mean that the proposed algorithm is implemented over the traffic data obtained in traffic gateway system. The traffic data eventually grows over progress of time and hence becomes massive challenging to be processed. In order to perform an effective decision for navigational system, it is required that data should be crisp and non-redundant that will offer accurate and faster decision making of an effective route exploration. The study considers that these signals generated by the visual sensors are tagged with GPS-based information as a part of geo-referencing. For this reason, the signal becomes quite heavy and it is required to minimize the dimension of the signal before processing. Therefore, the prime purpose of this algorithm is extract, process, and reduce the obtained traffic signal in order to make it suitable for further processing. The algorithm takes the input of the visual sensor data $d$ from the traffic gateway system and extracts information associated with number of traffic $T_{n}$ and position of traffic $T_{p}$ (Line-1). This information $\left(T_{n} T_{p}\right)$ is termed as geo-referenced metadata. A traffic object of location Tloc is constructed after reading this metadata (Line-3). In similar fashion, the algorithm extracts information associated with streamed data from the gateway using $\mathrm{T}_{\mathrm{n} 1}$ and $\mathrm{T}_{\mathrm{p} 1}$ and constructs a location object $\mathrm{T}_{\text {loc1 }}$ (Line-4). The algorithm performs certain property fine tuning by signifying the range of the test signal test_signal obtained from $\mathrm{T}_{\text {loc }}$ considering specific number of signal $\left(\mathrm{n}_{\text {signal }}\right)($ Line-6). For quantization of signal, the algorithm considers test signal $\mathrm{T}_{\text {signal }}$ (Line-7) and carry out further computation process. A signal sig is obtained after reading the digitized signal extracted from $\mathrm{T}_{\text {loc }}$ (Line-8) and then it is further subjected to a resizing function $f(\mathrm{x})$ for signal $\operatorname{sig}_{2}$ obtained from prior step (Line-9). Apart from this, the function $f(\mathrm{x})$ also carries out size control and therefore the obtained signal is reduced in its size. Finally, the obtained signal with respect to streamed data of traffic $T_{\text {loc1 }}$ is overwritten on top of reduced signal of traffic sig (Line-10). The processing of this algorithm finally results in output signal $\mathrm{o}_{\text {sig }}$ (Line-10) which is dimensionally reduced in size. To highlights the process flow of the proposed algorithm. The steps of algorithm are: 


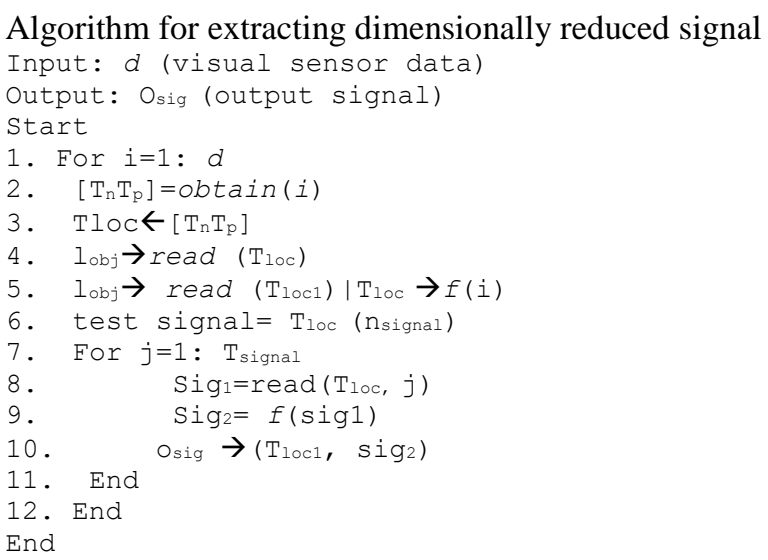

The contributions of this algorithm are; i) it assists in dimensional reduction which is network friendly for transmission, ii) it initiates a proper indexing mechanism with respect to number of vehicles and respective location of them, and iii) it is free from any ambiguous processing as all information are maintained in form of matrix.

\subsubsection{Algorithm for traffic feature extraction}

After the output signal, which is dimensionally reduced, is obtained, the next step is to extract highly informative contents from it. However, owing to streaming nature of the geo-referenced traffic signal, it is challenging to extract unique information out of it. Therefore, the proposed system carries out the extraction of significant feature from the location object information so that navigational decision can be carried out over highly refined data in the form of features. Conventionally, the size of feature is very much less compared to the original traffic data considered. Therefore, the prime responsibility of this algorithm is to perform extraction of significant traffic related information. The steps of the algorithm are as follows:

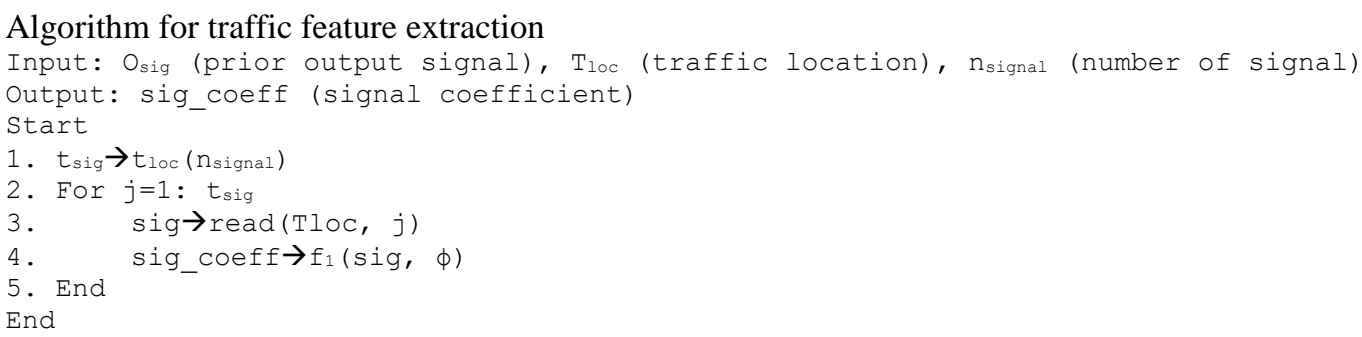

The algorithm takes the input of priory output signal $\mathrm{O}_{\text {sig }}$ from this similar number $\mathrm{T}_{\mathrm{n} 2}$ and position $\mathrm{T}_{\mathrm{p} 2}$ information are constructs a new location object $\mathrm{T}_{\text {loc2 }}$ followed by construction of a signal structure $n_{\text {signal }}$ consisting of number of signals, dimension of traffic signal. Initially, the algorithm obtains a test signal $t_{\text {sig }}$ from $n_{\text {signal }}$ mapped with traffic location object $t_{\text {loc }}$ (Line-1). Considering all the test signal $t_{\text {sig }}$ (Line-2), the algorithm obtains signal with respect to location object $\mathrm{T}_{\text {loc }}$ (Line-3) followed by extraction of signal coefficient (Line-4). The algorithm implements a special function $\phi$ which is responsible for carrying out explicit operation where the dominant signal information in the signal distribution data is obtained. A specific thresholding is carried out to extract the dominant signal data. The information carried out by the visual sensor data are therefore filtered to higher degree and only explicit features are obtained in the form of signal coefficient (Line-4). A function $f_{1}(\mathrm{x})$ is constructed for this purpose where the location object $\mathrm{T}_{\text {loc } 2}$ is overwritten on top of signal obtained in prior step sig (Line-4) as an outcome. To highlights the process flow for similar algorithmic steps. The contribution of this algorithms are: i) this algorithm further contributes to size reduction where the data are now replaced to signal coefficient (i.e. feature); ii) the accuracy is vehicle identification with respect to number and position is further enhanced in the form of interactive block of operation between the extraction of feature and construction of new object; iii) the algorithm also contributes towards lowering memory dependency and controlling of time consumption in traffic stream.

\subsubsection{Algorithm for mobile object tracking}

In traffic system, vehicle is considered as a mobile object and proposed system aims for exploiting the geo-referenced sensory data for making a decision. This decision should eventually lead to a precise identification of an alternative route in autonomous navigation system. The idea leads to precise tracking of all the vehicle leading to better decision. The steps of the algorithm are as follows: 


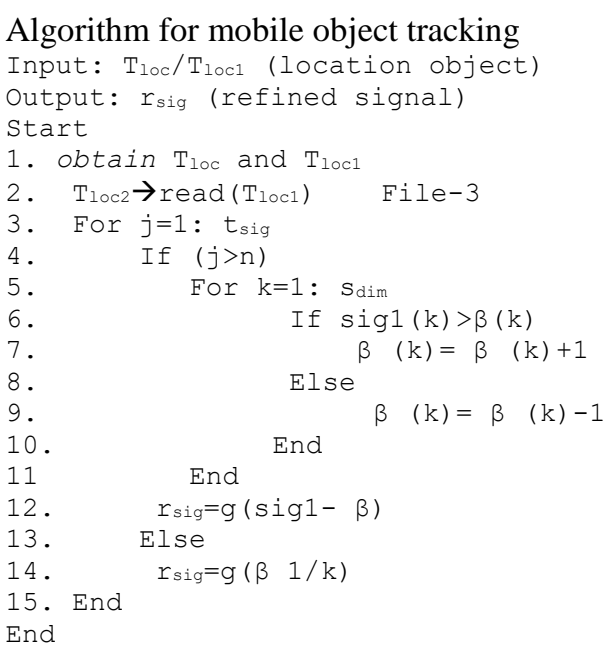

The core idea of this algorithm is to process it using inputs of location objects obtained in prior steps in order to yield an outcome of refined signal. The proposed system initially takes location object of $\mathrm{T}_{\text {loc }}$ and $\mathrm{T}_{\text {loc1 }}$ which is followed by further extraction of another location object $\mathrm{T}_{\text {loc2 } 2}$ obtained from $\mathrm{T}_{\text {loc } 1}$ itself (Line-1 and Line-2). For all the considered test signal $t_{\text {sig }}$ (Line-3) as well considering all the dimension of signal $s_{\text {dim }}$ (Line-5), the algorithm applies a first logical condition where signal $\mathrm{sig}_{1}$ is compared with contextual matrix $\beta$ (Line-6). Basically, $\beta$ is a two dimensional matrix and is constructed over same size equivalent to dimensional information of the signal. The signal $\operatorname{sig}_{1}$ is basically dimensionally reduced version of digitized location object $\mathrm{T}_{\text {loc. }}$. The first logical condition (Line-6) leads to generation of values to be filled in contextual matrix $\beta_{1}$ which is equivalent to expression written in Line-7. Otherwise, it leads to different expression shown in Line-9. This yield of $\beta$ is further finalized by applying precision function $g(\mathrm{x})$ over value obtained by subtracting sig1 from contextual matrix $\beta$ (Line-12). This leads to generation of refined signal value. However, this value is applicable for the condition $j>n$ (Line-4) otherwise, the different value of the $\beta$ given by the expression highlighted in Line-14 of algorithm using similar precision function $g(\mathrm{x})$. The contributions of this algorithm are i) it offers a precise tracking of the mobile object from this refined signal, ii) it is flexible to include various logical condition as per the traffic scenario, and iii) it offers faster computation as the complete execution is done over traffic object and not the raw data.

\section{RESULTS AND DISCUSSION}

This section discusses about the outcome obtained from the evaluation of the proposed system. The implementation of the proposed model is carried out over traffic surveillance dataset.

\subsection{Dataset used}

The proposed system takes the input to the visuals captured from the sensors deployed in the traffic region which are also geo-referenced. For this purpose, it is essential to select such a dataset which is shot at specific location that can be easily captured from GPS. The proposed system makes use of publicly available dataset [32] where various signals are maintained in the form of video and they are also tagged programmatically with specific traffic location of GPS information. Following is the detailed information about the dataset.

The analysis has been carried out considering 5 different types of datasets. The first dataset is Sherbrook which is characterized by $800 \times 600$ resolution of video while the analysis can be easily carried out for 30 frames per second for the event scene that is selected with 5 numbers of pedestrian and 15 vehicles. The interesting part of the scene is that it has 7 moving objects at same time. The second dataset considered is Rouen which has $1024 \times 576$ resolution of video. The assessment can be carried out for 25 frames per second considering 11 number of pedestrians, single motorcycle, and 4 moving vehicles and all of them are annotated. The third dataset is St-Marc which has better resolution of video as 1280x720. The scene consists of 30 frames per second with 19 numbers of pedestrian, 2 motorcycle, and 7 vehicles while 14 numbers of objects are found to simultaneously move. The fourth dataset is Jinhua city which is characterized by $1280 \times 720$ video resolutions. The uniqueness of this video is its presence of large number of vehicles compared to any other dataset in a much narrowed lane. Figure 2 (a)-(d) shows how an ambulance that runs at the middle of lane trying to cross it. All these videos are programmatically tagged with geo-referenced data from GPS and hence solve a better purpose of analysis. 


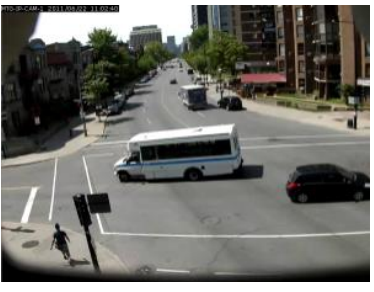

(a)

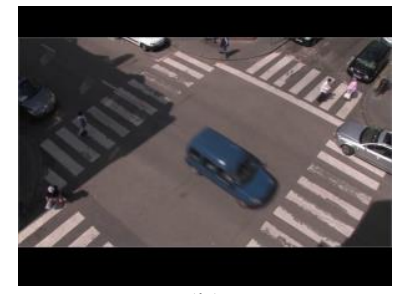

(b)

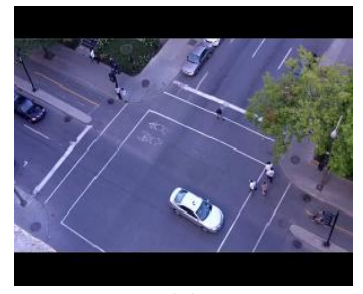

(c)

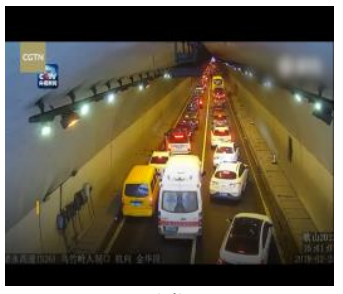

(d)

Figure 2. Snapshots of the dataset used [32]; (a) Sherbrook, (b) Rouen, (c) St-Marc, and (d) Jinhua City

\subsection{Visual outcome of study}

The direct visual signals that are captured are subjected to all the 3 algorithms to find that there are simultaneous computations of tracking all the vehicles on the field of event in dataset followed by different options of routes for the given scene in the form of numerical coordinates. The outcomes of visuals are quite distinct and can be easily evaluated with respect to alternative routes explored. It is assumed that these alternative routes coordinates are then forwarded to the vehicles with certain communication medium and the vehicles are anticipated to follow the new routes in their inbuilt navigation system. The visual outcomes are shown in Figure 3.
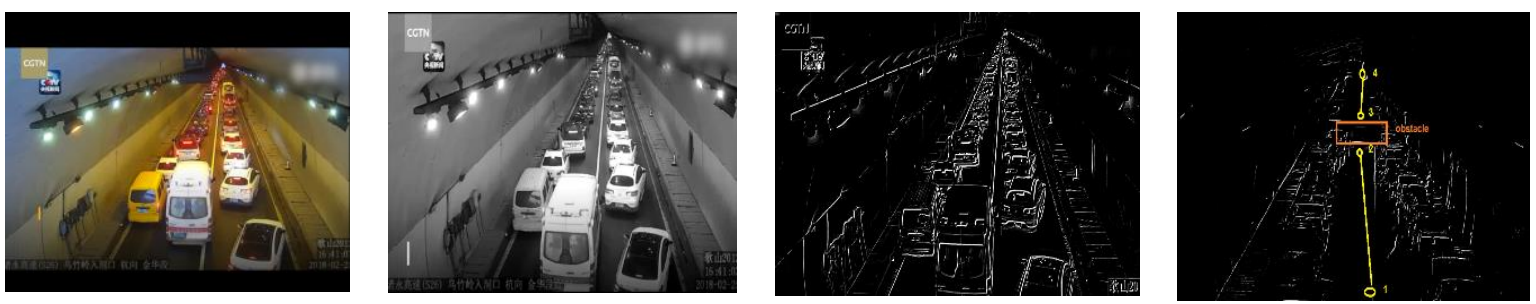

Figure 3. Visual outcomes of signal extracted from visual sensor

However, there are also possibilities of any form of disparity occurring between the images that are geo-referenced and the directional vector of an alternative route. For this purpose, the proposed system initially selected 25 different points over the moving vehicles in order to offer if the counting of the vehicles as well as their respective position matches for the given traffic scene. The deployed points are then subjected to MATLAB where they are superimposed on the top of the signal captured from visual sensors $\left(\mathrm{T}_{\text {loc }}\right)$ as well as they also superimpose the stream of the image which has GPS based location outcome $\left(\mathrm{T}_{\text {loc1 }}\right)$. The system then explores the presence of any scene that corresponds to road or lane which are obtained after background subtraction using contextual matrix $\beta$. The idea is basically to differentiate the vehicles and the route / lane so that better form of alternative path can be identified with respect to variable directions of vehicles. By doing this operation of superimposing the vehicles from the signal and the explored points, the system gives different forms of discrepancies with respect to the position. In such case, the fluctuation of the shift direction of a vehicle (whose directionality is undefined at specific instant of time), it is quite clear that proposed system will not be able to perform complete matching of an image directionality of a vehicle to the destination point on alternative routes. In order to prevent this problem, it is required to find out any such form of an error between all the directionality vectors. However, it should be also known that such kind of difference (or error/variation) is also on the basis of the user in order to find out correct alternative path and correct count of vehicles. It is required to be done as the proposed system offers navigational system on the basis of signals in the form of an image which has higher feasibility of inconsistencies as well as errors incorporated by the human itself. Although, it is feasible to perform recalibration of the signals obtained from traffic gateway about the image with respect to some standard, but it will be highly expensive aspect for majority of the user.

The proposed system makes use of World Geodetic system also known as WGS84 as a standard coordinate system that is used for geo-referencing over the receiver of GPS system. Proposed system does not intend to use any form of the mathematical approach of transformation as it would result in faulty outcome. Another reason is that GPS highlights the location variation for a single location for each consecutive date irrespective of any selection of the coordinate system. Therefore, adoption of any form of spatial shift operation in geo-referenced data could offer potential enhanced accuracy. Apart from this, it will 
also maintain a cost effective computational operation by significantly controlling any form of computational complexity. Hence, the proposed system offers finely-tuned location information which offers higher accuracy for a given instance of geographic region as well as exact time in that location. A manual assessment of 5 hours is carried out over this data iteratively to find out that it offers better location stability. A closer look in this outcome also shows that proposed system offers a user-friendly outcome when it comes to navigational support using both computation vision and GPS based information. The relay of the stream is also reduced in data size for which reason the proposed system also offers light-weight traffic data transmission over network where decision towards traffic-new routes can be almost instantaneously stated to be adopted and followed.

\subsection{Numerical outcome of study}

The numerical outcome of the proposed study is assessed with respect to two performance parameters i.e., i) accuracy and ii) mean processing time. The outcomes discussed over here are obtained after testing with all the 5 dataset and obtaining a mean value of them. Apart from this, the study outcome is compared extended Kalman filter, which is reported to be extensively used in existing system of navigational system.

From Figure 4, it can be seen that proposed system offers better positional accuracy in contrast to existing Kalman filter. The rationale of this outcome is-basically extended Kalman filter can perform optimal estimation if there is a presence of non-linearity in the data obtained from scene. Therefore, there is no difference in operation in extended Kalman filter with its legacy version if there existing linear state transition model. The proposed system offers $95.21 \%$ of accuracy while existing system offers $82.368 \%$ of accuracy. This is not a case in majority of practical traffic application.

From Figure 5, it can be seen that proposed system offers highly reduced processing time in comparison to extended Kalman filter. Non-inclusion of any iterative operation and proper indexing of scene (vehicle) leads to faster yield of counting of vehicles, tracking them, and exploring alternative routes. However, extended Kalman filter offers highly iterative operation, which could lead to consumption of time. Proposed system takes only 0.0401 seconds while existing system takes 0.7498 seconds for processing.

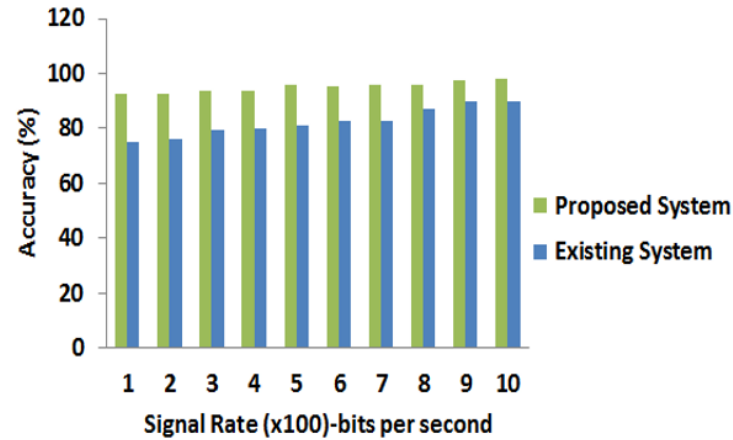

Figure 4. Comparative analysis of positional accuracy

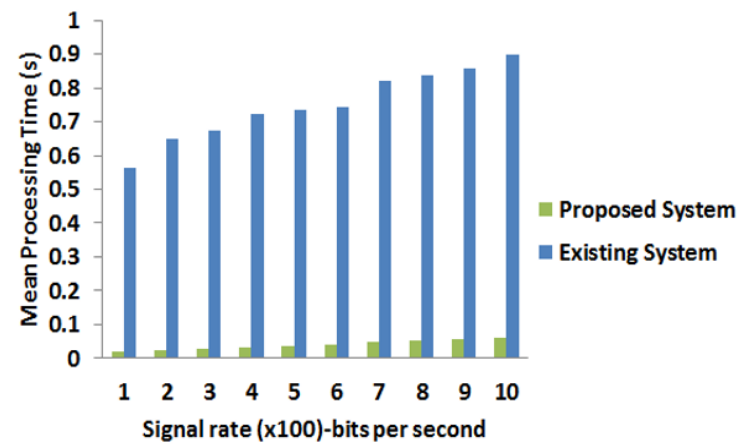

Figure 5. Comparative analysis of mean processing time

\section{CONCLUSION}

This paper has presented a novel approach of navigation system where joint implementation of GPS and computer vision is used. An analytical model is designed for this purpose where the signals captured from visual sensors are used as an input. The study considered that these signals are geo-referenced using GPS data which are then forwarded autonomously from vehicle to traffic gateway system where the proposed algorithm is considered to be deployed. The algorithm performs sequential steps of operation in order to process the signal leading to outcome of instantaneous capturing of the traffic over the scene. The contribution of the proposed system are as follows: i) the proposed system is a computational model that can be applicable for any form of visually captured signals, ii) the algorithm is non-iterative and hence it is faster and progressive in its operation leading to cost effectivity, iii) the model is found to offer approximately $12 \%$ increment in accuracy compared to frequently implemented Kalman filter and ii) the model offers faster processing time where the improvement is found to be approximately $70 \%$ better than existing approach. The future work will be continued towards achieving further optimization-based approaches to offer more improvement considering new challenges. 


\section{REFERENCES}

[1] R. B. Rustamov, S. Hasanova, and M. H. Zeynalova, "Multi-purposeful application of geospatial data," IntechOpen, 2018, doi: 10.5772/intechopen.69713

[2] A. Hussain, H. Magsi, A. Ahmed, H. Hussain, Z. H. Khand, and F Akhtar, "The effects of using variable lengths for degraded signal acquisition in GPS receivers," International Journal of Electrical and Computer Engineering (IJECE), vol. 11, no. 4, pp. 3201-3211, 2021, doi: 10.11591/ijece.v11i4.pp3201-3211.

[3] A. A. A. Solyman and I. A. Elhaty, "Potential key challenges for terahertz communication systems," International Journal of Electrical and Computer Engineering (IJECE), vol. 11, no. 4, pp. 3403-3409, 2021, doi: 10.11591/ijece.v11i4.pp3403-3409.

[4] D. U. Sanli, "Accuracy of GNSS Methods," IntechOpen, 2019, doi: 10.5772/intechopen.75424.

[5] M. Golio and J. Golio, "RF and microwave applications and systems," CRC Press, 2008.

[6] N. M. Vani and S. Banu, "Evaluation of GPS data for navigational solution and error reduction using kalman filter," 2018 International Conference on Design Innovations for 3Cs Compute Communicate Control (ICDI3C), Bangalore, 2018, pp. 12-17, doi: 10.1109/ICDI3C.2018.00012.

[7] M. L. Cherif, J. Leclère and R. J. Landry, "Loosely coupled GPS/INS integration with snap to road for low-cost land vehicle navigation: EKF-STR for low-cost applications," 2018 IEEE/ION Position, Location and Navigation Symposium (PLANS), 2018, pp. 275-282, doi: 10.1109/PLANS.2018.8373391.

[8] H. Ko, B. Kim and S. Kong, "GNSS multipath-resistant cooperative navigation in urban vehicular networks," in IEEE Transactions on Vehicular Technology, vol. 64, no. 12, pp. 5450-5463, Dec. 2015, doi: 10.1109/TVT.2015.2481509.

[9] R. Prabha and Mohan G. Kabad, "A comprehensive insight towards pre-processing methodologies applied on GPS data," International Journal of Electrical and Compputer Engineering (IJECE), vol. 10, no. 3, pp. 2742-2754, 2019, doi: 10.11591/ijece.v10i3.pp2742-2754.

[10] A. Abosekeen, A. Noureldin and M. J. Korenberg, "Improving the RISS/GNSS land-vehicles integrated navigation system using magnetic azimuth updates," in IEEE Transactions on Intelligent Transportation Systems, vol. 21, no. 3, pp. 1250-1263, Mar. 2020, doi: 10.1109/TITS.2019.2905871.

[11] Y. Guo, M. Wu, K. Tang, J. Tie and X. Li, "Covert spoofing algorithm of UAV based on GPS/INS-integrated navigation," in IEEE Transactions on Vehicular Technology, vol. 68, no. 7, pp. 6557-6564, Jul. 2019, doi: 10.1109/TVT.2019.2914477.

[12] F. Li and L. Chang, "MEKF with navigation frame attitude error parameterization for INS/GPS," in IEEE Sensors Journal, vol. 20, no. 3, pp. 1536-1549, 2020, doi: 10.1109/JSEN.2019.2947456.

[13] M. Z. Mostafa, H. A. Khater, M. R. Rizk and A. M. Bahasan, "GPS/DVL/MEMS-INS smartphone sensors integrated method to enhance USV navigation system based on adaptive DSFCF," in IET Radar, Sonar and Navigation, vol. 13, no. 10, pp. 1616-1627, 10 2019, doi: 10.1049/iet-rsn.2019.0015.

[14] L. Steiner, M. Meindl, C. Marty and A. Geiger, "Impact of GPS processing on the estimation of snow water equivalent using refracted GPS signals," in IEEE Transactions on Geoscience and Remote Sensing, vol. 58, no. 1, pp. 123-135, Jan. 2020, doi: 10.1109/TGRS.2019.2934016

[15] G. Wang, X. Xu, Y. Yao, and J. Tong, "A novel BPNN-based method to overcome the GPS outages for INS/GPS system," in IEEE Access, vol. 7, pp. 82134-82143, 2019, doi: 10.1109/ACCESS.2019.2922212.

[16] Y. Zhang, "A fusion methodology to bridge GPS outages for INS/GPS integrated navigation system," in IEEE Access, vol. 7, pp. 61296-61306, 2019, doi: 10.1109/ACCESS.2019.2911025.

[17] D. Li, X. Jia, and J. Zhao, "A novel hybrid fusion algorithm for low-cost GPS/INS integrated navigation system during GPS outages," in IEEE Access, vol. 8, pp. 53984-53996, 2020, doi: 10.1109/ACCESS.2020.2981015.

[18] Q. Guo, W. H. Deng, O. Bebek, M. C. Cavusoglu, C. H. Mastrangelo and D. J. Young, "personal inertial navigation system assisted by MEMS ground reaction sensor array and interface ASIC for GPS-denied environment," in IEEE Journal of Solid-State Circuits, vol. 53, no. 11, pp. 3039-3049, Nov. 2018, doi: 10.1109/JSSC.2018.2868263.

[19] J. Dong, M. Noreikis, Y. Xiao and A. Ylä-Jääski, "ViNav: a vision-based indoor navigation system for smartphones," in IEEE Transactions on Mobile Computing, vol. 18, no. 6, pp. 1461-1475, Jun. 2019, doi: 10.1109/TMC.2018.2857772.

[20] G. Lentaris, I. Stratakos, I. Stamoulias, D. Soudris, M. Lourakis and X. Zabulis, "High-performance vision-based navigation on SoC FPGA for spacecraft proximity operations," in IEEE Transactions on Circuits and Systems for Video Technology, vol. 30, no. 4, pp. 1188-1202, Apr. 2020, doi: 10.1109/TCSVT.2019.2900802

[21] A. Manzanilla, S. Reyes, M. Garcia, D. Mercado, and R. Lozano, "Autonomous navigation for unmanned underwater vehicles: real-time experiments using computer vision," in IEEE Robotics and Automation Letters, vol. 4, no. 2, pp. 1351-1356, Apr. 2019, doi: 10.1109/LRA.2019.2895272.

[22] L. Zhang, Z. Zhai, L. He and W. Niu, "Infrared-based autonomous navigation for civil aircraft precision approach and landing," in IEEE Access, vol. 7, pp. 28684-28695, 2019, doi: 10.1109/ACCESS.2019.2893062.

[23] R. P. Padhy, F. Xia, S. K. Choudhury, P. K. Sa, and S. Bakshi, "Monocular vision aided autonomous UAV navigation in indoor corridor environments," in IEEE Transactions on Sustainable Computing, vol. 4, no. 1, pp. 96-108, 1 Jan.-Mar. 2019, doi: 10.1109/TSUSC.2018.2810952

[24] C. Lee and D. Kim, "Visual homing navigation with haar-like features in the snapshot," in IEEE Access, vol. 6, pp. 33666-33681, 2018, doi: 10.1109/ACCESS.2018.2842679.

[25] H. Xiong, Z. Mai, J. Tang and F. He, "Robust GPS/INS/DVL navigation and positioning method using adaptive federated strong tracking filter based on weighted least square principle," in IEEE Access, vol. 7, pp. 26168-26178, 2019, doi: 10.1109/ACCESS.2019.2897222.

[26] L. Cheng, B. Song, Y. Dai, H. Wu, and Y. Chen, "Mobile robot indoor dual kalman filter localisation based on inertial measurement and stereo vision," in CAAI Transactions on Intelligence Technology, vol. 2, no. 4, pp. 173-181, 2017, doi: 10.1049/trit.2017.0025.

[27] R. Prabha and M. G. Kabadi, "KNODET: a framework to mine gps data for intelligent transportation systems at traffic signals," 2017 International Conference on Recent Advances in Electronics and Communication Technology (ICRAECT), 2017, pp. 85-89, doi: 10.1109/ICRAECT.2017.58.

[28] Z. Zhu and C. Taylor, "Conservative uncertainty estimation in map-based vision-aided navigation," in IEEE Transactions on Aerospace and Electronic Systems, vol. 53, no. 2, pp. 941-949, Apr. 2017, doi: 10.1109/TAES.2017.2667278.

[29] L. Li, Q. Xu, V. Chandrasekhar, J. Lim, C. Tan and M. A. Mukawa, "A wearable virtual usher for vision-based cognitive indoor navigation," in IEEE Transactions on Cybernetics, vol. 47, no. 4, pp. 841-854, Apr. 2017, doi: 10.1109/TCYB.2016.2530407.

[30] J. M. Prendergast, G. A. Formosa, and M. E. Rentschler, "A platform for developing robotic navigation strategies in a deformable, dynamic environment," in IEEE Robotics and Automation Letters, vol. 3, no. 3, pp. 2670-2677, Jul. 2018, doi: 10.1109/LRA.2018.2827168. 
[31] R. Prabha, and K. G. Mohan, "PPT-NS: prioritized progressive trend based navigational system on GPS data with artifacts," International Journal of Innovative Technology and Exploring Engineering, vol. 9, no. 2, Dec. 2019, doi: 10.35940/ijitee.B6554.129219.

[32] G. Velasco-Hernández, "Traffic-surveillance-dataset," Github, Accessed: Jul. 13, 2020. [Online]. Available: https://github.com/gustavovelascoh/traffic-surveillance-dataset

\section{BIOGRAPHIES OF AUTHORS}

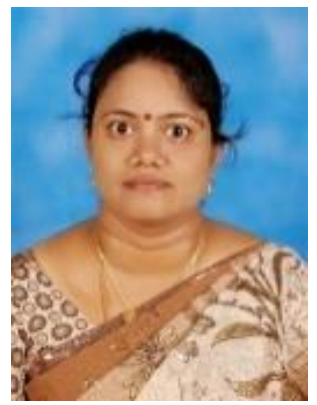

Prabha Ramasamy (D) If SC P pursued Bachelor of Computer Science and Engineering from Madurai Kamarajar University, 1997. Completed Master of Technology in Computer Science and Engineering from Visvesvaraya Technological University, Belagavi in 2010. She is currently working at Nettur Technical Training Centre, Bangalore as an Assistant Manager and pursuing Ph.D from Visvesvaraya Technological University. Her research focuses on IoT, machine learning and deep learning. She has 17 Years of teaching experience and 4 years of research experience. She can be contacted at email: research.prabha.r@gmail.com.

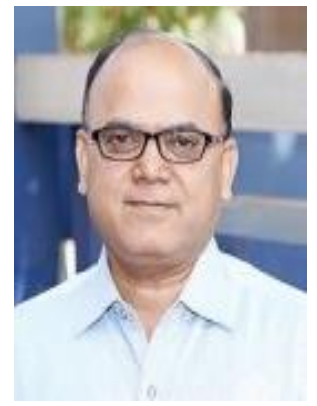

Mohan Kabadi (D) 8d SC P received Bachelor Degree in Electrical Engineering from University of Mysore in 1984 and Master of Technology in Power and Energy System from KREC (Mangalore University) during 1995. Received Ph.D with the specialization of computer architecture from Anna University in the year 2007. He has published and presented more than 30 research papers in reputed International Journals and Conferences. His area of research includes low power architecture design, wireless sensor networks, IoT, cloud computing, network security and cryptography. He has 30 years of teaching experience and 14 years of research experience. He can be contacted at email: mohankg@presidencyuniversity.in. 